\title{
2163. Method for studying the structure of blast-induced vibrations in open-cast mines
}

\author{
Józef Pyra ${ }^{1}$, Anna Soltys ${ }^{2}$ \\ AGH University of Science and Technology, Cracow, Poland \\ ${ }^{1}$ Corresponding author \\ E-mail: ${ }^{1}$ pyra@agh.edu.pl, ${ }^{2} a n n a . s o l t y s @ a g h . e d u . p l$ \\ Received 8 April 2016; received in revised form 14 July 2016; accepted 18 August 2016 \\ DOI http://dx.doi.org/10.21595/jve.2016.17052
}

\begin{abstract}
Detonations of explosive material charges are a source of intense paraseismic vibrations. These vibrations can cause damage to buildings and other infrastructure in the vicinity of the works, and can be a nuisance for their inhabitants. The article presents the problem of paraseismic vibration analysis, as induced by blasting works carried out in open-cast mines, using various methods of time - frequency, providing information about the duration of the signal, the frequency structure, and its energy.
\end{abstract}

Keywords: signal analysis, paraseismic vibrations, matching pursuit, impact of vibration on the environment.

\section{Introduction}

Explosives are used in the military and civilian industries, such as mining, geology, construction, and the road industry.

The effective use of explosive materials will always induce vibrations propagated around the place of work. We should be under no illusions in this regard. However, not all vibrations propagated in the environment and transferred to buildings are harmful and disturbing for inhabitants.

Minimizing the impact is achieved through preventive actions, based primarily on the knowledge of the technology of the conducted blasting works, as well as on more accurate diagnosis of the nature of vibration waves spreading around, by assessing their impact on facilities in the vicinity.

In most cases, the use of a relevant scale is a sufficient method of assessing the harmfulness of vibrations transmitted through the ground into buildings. The first scales were created to assess the effects of earthquakes - natural seismic activity. Despite delivering a series of works aimed at adjusting these scales in order to assess the impact of mining shocks, satisfactory results have not been achieved, due to significant differences in the characteristics of induced vibrations [1]. Even greater differences exist in the case of vibrations induced during the works carried out with explosive materials. Using explosive materials, for example, in the process of rock material exploitation, causes vibrations in the rock environment, vibrations with relatively high frequency and short duration. These two parameters, frequency and duration, associated with the vibration source, can be the basis for a clear division between the phenomena associated with earthquakes, mining shocks and vibrations resulting from the use of explosive materials in mines and engineering works.

As already mentioned, assessment of the impact of vibrations induced by blasting works is performed using scales. For example, Fig. 1 shows: the SWD-I scale used in Poland (in accordance with PN-B-02170:1985 [2]) and the American scale developed by the Bureau of Mines. Both cases show the parameters to be assessed are the velocity and frequency of vibration.

Fig. 1 also shows the importance of the frequency of vibrations in assessments. In order to illustrate this problem, Fig. 2 shows the SWD I scale with limit values for vibration velocity, taking the boundary B as an acceptable level of vibration intensity. For example, frequencies $25 \mathrm{~Hz}, 10 \mathrm{~Hz}, 5 \mathrm{~Hz}$ and $2 \mathrm{~Hz}$ were selected. The acceptable values of velocity are, respectively: $0.62 ; 1.6 ; 3.0$ and $50.0 \mathrm{~mm} / \mathrm{s}$ [3]. The data shows the difference of vibration intensity is very large 
-80 times for extreme frequencies.
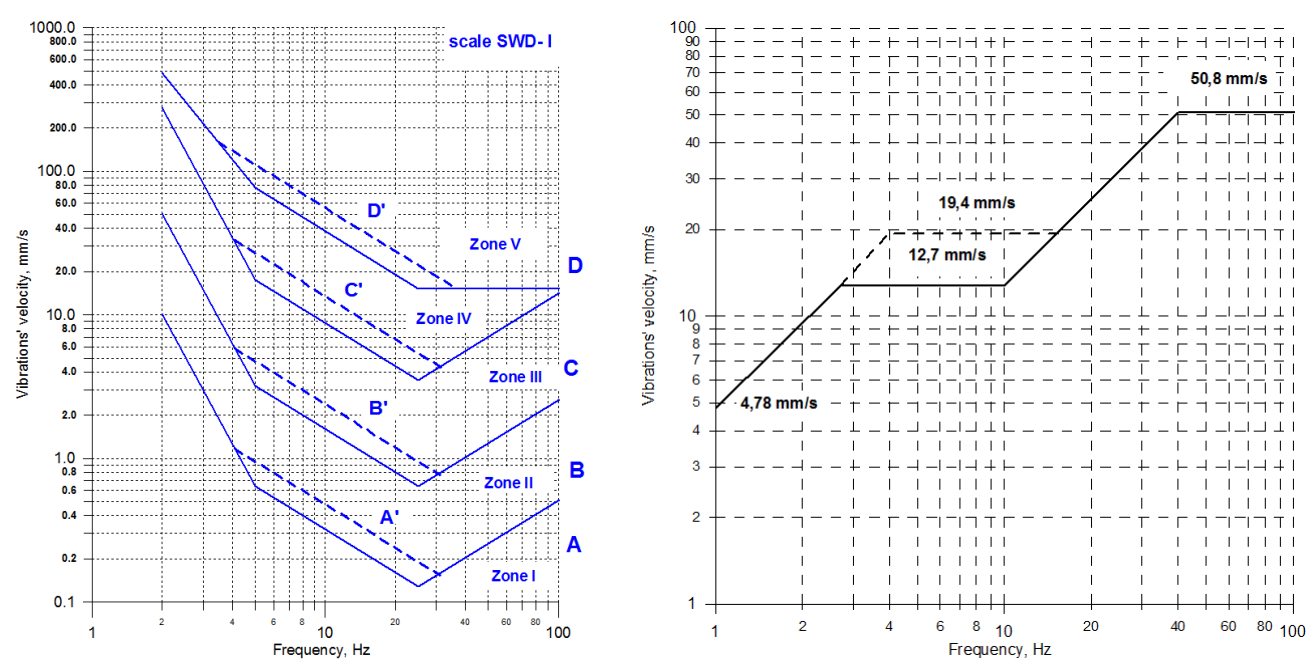

Fig. 1. The SWD-I scale and the scale of harmfulness according to the Bureau of Mines $[2,4]$

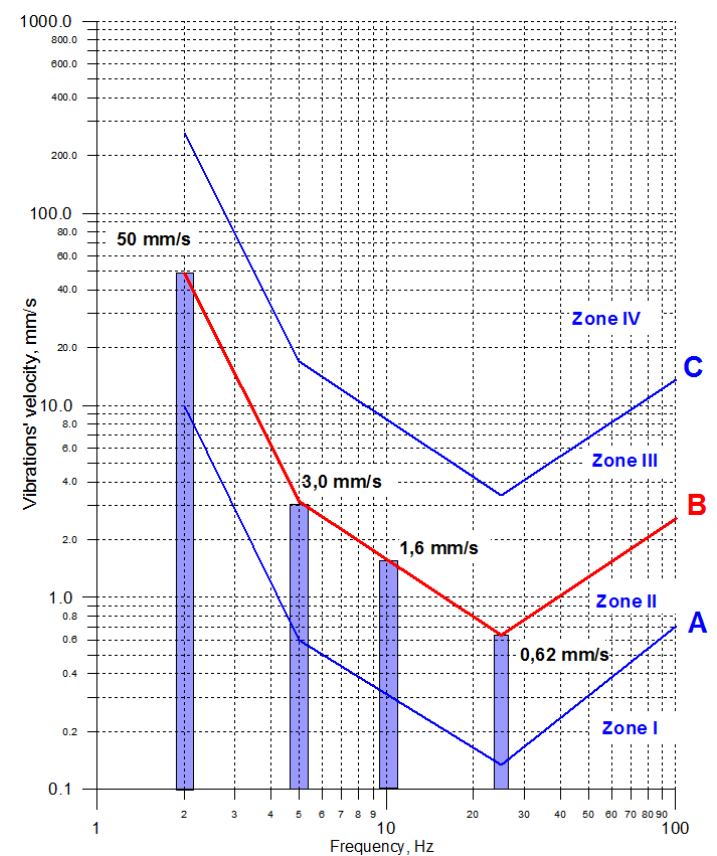

Fig. 2. SWD I scale - characteristic velocity limit values for frequencies of $25,10,5$ and $2 \mathrm{~Hz}[3]$

Numerous studies suggest [5-8] that a millisecond delay significantly affects the structure of vibrations induced while blasting works, which means that the proper selection of the millisecond delay can change the dominant frequencies. Following this path, a frequency shift of $10 \mathrm{~Hz}$ in the range of $25 \mathrm{~Hz}$ changes the qualification of vibrations from Zone II to Zone III, assuming the same intensity of vibration.

Changing the vibration frequency caused by the applied delay is an element which rarely receives attention when designing blasting works.

In most cases, impact assessment using the scales (Fig. 1) refers to measurements performed on the foundations of protected areas, and yet, vibrations induced by detonating explosive 
materials are propagated through the ground, meaning that the phenomenon of building-ground interaction is a very important element of the research, as the way of transferring vibrations from the ground to the facility is largely dependent on the frequency of vibrations occurring in the ground. It can be assumed that greater vibration frequency is increasingly suppressed during the transfer from the ground to the building while vibrations with lower frequencies may not be modified. This means that the phenomenon of building-ground interaction substantially depends on the oscillation of frequency and should be taken into account in testing procedures.

Paraseismic vibrations induced as a result of detonation of explosive materials belong to a group of non-stationary signals. Their structure is variable (folded), consisting of a whole series of harmonics, making any analysis and assessment of the impact of these types of vibrations on the environment a complex process. It requires consideration of many factors that may affect the intensity of vibrations, and finding the best solution to enable acquiring accurate knowledge of the essence of vibrations propagated to the environment.

Until now, several methods of analysis have been used for signals, which more or less reflect the nature of the blast-induced vibrations and the possibility of their detrimental effect on the environment. The amplitude, filter, and spectral analysis methods may be mentioned here.

\section{Digital recording of vibrations as a signal of parameter variation over time - signal analysis}

Paraseismic vibrations can be described as signals varying in time and carrying information about the nature of the studied phenomenon associated with detonating explosive materials. Among the various classifications of signals, paraseismic vibrations are in the group of non-stationary random transient processes [9]. They may contain a number of non-stationary transfer characteristics, such as slow or sudden changes, or pulse-like characteristics. These are transients under which impulse energy transfers take place to the oscillating element in the signal transduction (in the case of detonations of explosive materials, vibrations propagate in the rock), manifested by a sharp temporal increase in signal energy. In other words, paraseismic vibrations recorded in certain circumstances require a highly individual approach and detailed analysis in order to obtain as much information about their structure as possible [7].

Analyzing the technical literature, it can be concluded that it is most common to analyze signals as a function of time [10-13]. This type of analysis results in information about the maximum value of the measured parameter, for example: velocity (PPV). However, relying solely on the PPV value can result in overestimating the projected intensity of paraseismic vibrations, and thus lead to unnecessary restrictions on the permissible weight of explosive charges. Research shows that, in the case of such complex vibrations, the PPV value does not comprehensively describe their characteristics. The initiation systems currently available in mining allow blasting with large masses, spread over a longer time, which shows a significant impact the duration of the vibrations have on the degree of the resulting building hazard [14-16]. This means that analyses of vibration measurements should be carried out with regard to their duration, frequency and structure of the energy dissipated in the environment.

Taking these factors into account, there are several methods currently being used:

1) analysis in the frequency domain - Fourier transform (FT),

2) time signal filtration - 3rd octave band analysis,

3) short-time Fourier transform (STFT),

4) analysis in time and frequency domains - wavelet transform (Wavelet Transform) and newer:

5) Wigner - Ville Transformation (WVT),

6) analysis in time and frequency domains - matching pursuit algorithm (Matching Pursuit).

The above-mentioned types of analysis have their advantages, but also a number of deficiencies which, of course, do not disqualify the method, but certainly require further research into one that will result in precise and more reliable information about the structure of vibration 
studied.

\subsection{Fourier transform (FT)}

Analysis in the frequency domain (i.e. spectral analysis) enables more accurate knowledge of the structure of vibrations $[8,9]$. It involves decomposition of the original time series (signal) into simpler components, the sine and cosine functions of various frequencies, and determining which ones are most important in building the image of vibrations (Fig. 3).

The result of this process is also the so-called Fourier transform or the Fourier integral. It shows the amplitude spectrum, illustrating the dependency of amplitudes of all signal components on frequency, and enables transforming any analysis of a function described in the time domain into the frequency domain. The effect of Fig. 2 signal analysis is shown in Fig. 4.

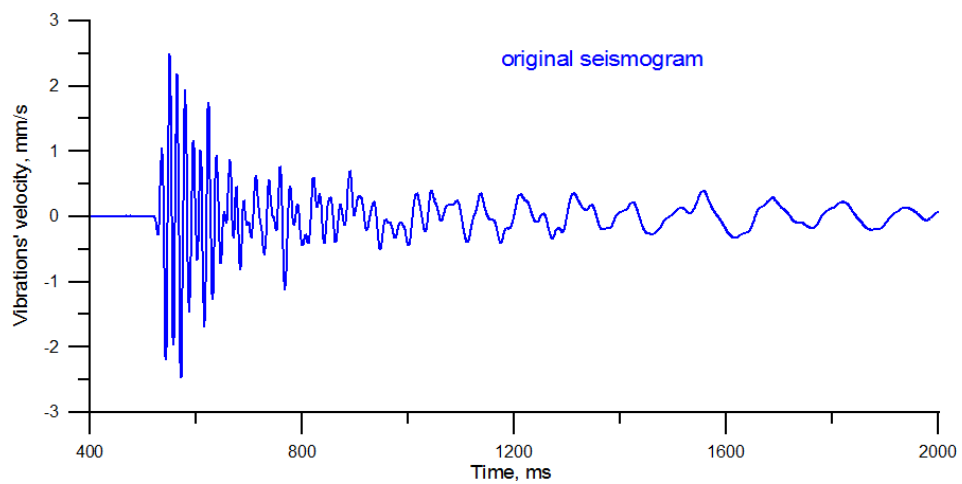

Fig. 3. The original recorded signal [7]

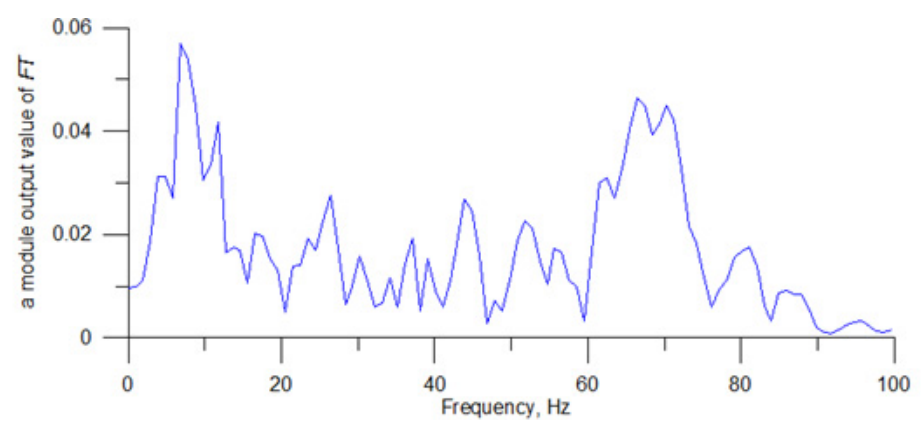

Fig. 4. Running time amplitude spectrum shown in Fig. 3 [7]

Unfortunately, signal analysis using the Fourier transform has a significant drawback - it shows the signal in the form of harmonic components, providing information on frequencies, but also losing information about the time of occurrence of the frequencies in the signal. In order to include the time domain signal, an inverse Fourier transform may be performed. That, however, loses data about the frequencies in the signal. It is, therefore, a method that does not give entirely satisfactory results.

Deficiencies of the analyses carried out, either in the time domain or in the frequency domain, contributed to the search for methods that can analyze both parameters simultaneously, giving fuller information on the frequency of the signal components and their occurrence within a specified period of time.

\subsection{3rd octave band analysis - time signal filtering}

One of the analyses providing information about the structure of vibrations both in the time 
domain and the frequency domain is filter analysis, carried out, among other things, by means of third octave filters $[8,17,18]$. As a result of the analysis, the measurement signal is distributed onto the components contained in certain frequency bands. The band-pass filter transmits only certain specific frequencies, suppressing those remaining.

Signal filtering by means of a set of band-pass filters enables division of the whole signal into smaller bands and analysis of the signal in a given frequency range. In the case of the 3rd octave band filtration, filters with a constant coefficient of bandwidth are used and the central frequency is a normalized value. As a result of such a filtration, one timeline results in a set of timelines, enabling a time analysis in the selected frequencies, which is not possible in the case of the Fourier transform (Fig. 5).
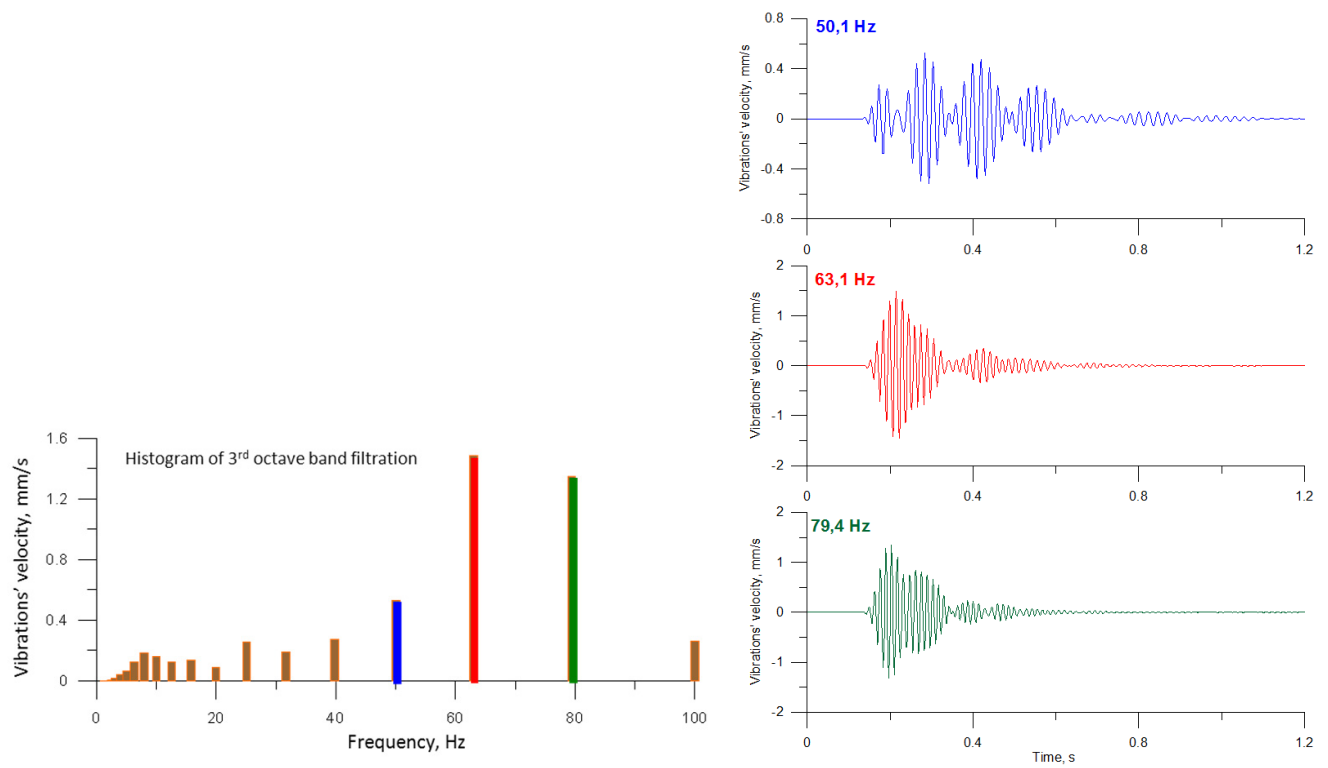

Fig. 5. An example of 3rd octave band filtration effect for the timeline course shown in Fig. 3 [7]

\subsection{Short-time Fourier transform (STFT)}

Another way of describing the structure of signals in the time domain and the frequency domain, much more precise than the filtration analysis, is the short-time Fourier transform (STFT) developed in 1946 by Dennis Gabor. He attempted to correct the imperfections of the Fourier transform and adapted it to the analysis of only a small portion of the signal in time [19]. This process is called windowing the signal. The STFT analysis involves cutting out successive sections of the signal using the window function and calculating the Fourier transform.

It should be noted, however, that the short-time Fourier transform provides information about when and which frequencies occurred at a given signal with limited precision. This limitation is due to the inconvenience of the STFT analysis, the once-adjusted width of the analysis window, the same for all frequencies. A wide window means a high frequency resolution but little time resolution. What is more, high frequencies are characterized in the time domain with the same precision. On the other hand, a window being too narrow makes it impossible to detect lower frequencies present in the signal. Unfortunately, the selection of the analysis window width is done by trial and error.

The result of the STFT Fig. 3 signal analysis is shown in Fig. 6.

Non-stationery signals are of a very different nature and, therefore, require a more flexible approach, in order to obtain accurate information in either the time domain or the frequency domain. This is enabled by another method of signal processing, the wavelet transform. 


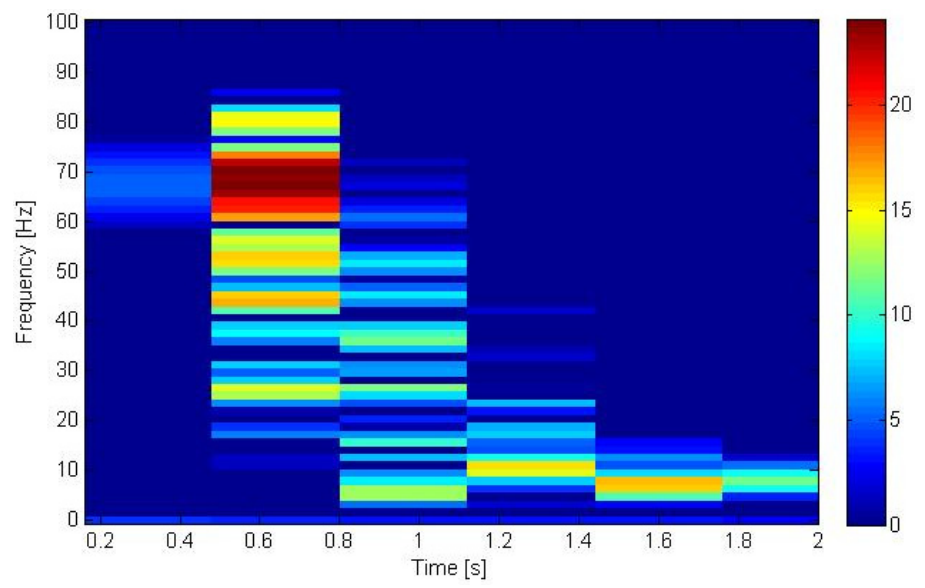

Fig. 6. The STFT analysis result for the time signal shown in Fig. 3 [7]

\subsection{Wavelet transform}

The wavelet transform is the next step in the analysis of signals, using the technique of windowing, but the analysis windows are of a different length and frequency [19, 20], which allows the use of long intervals when there is a need to capture more precise information about low frequencies and shorter areas where high frequency information is needed.

The wavelet transform represents a signal as a sum of the basic functions called wavelets, assuming a waveform of a limited duration, with the average value equal to zero. The wavelet feature is also a limited frequency band.

The wavelet is characterized by two basic parameters, i.e. the scale factor and the shift (translation). The scale factor changes the duration of wavelets, in other words, its compressions or tensions. In contrast, the translation factor provides the wavelet shift along the signal in the time domain, with a fixed scale factor, meaning a delay or acceleration of the beginning of the wavelet. Therefore, it is necessary to note that wavelet analysis does not include the time frequency area, but the time - scale area.

As a result of matching the wavelet to the signal, we obtain a number of CWT wavelet factors which determine the degree of correlation of the wavelets with the signal used (Fig. 7).

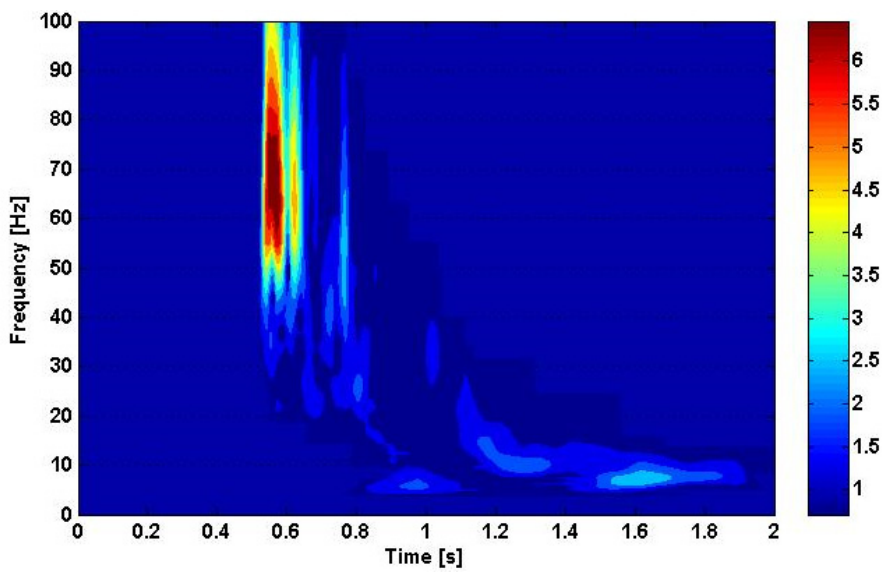

Fig. 7. Map of CWT wavelet factors for the time signal shown in Fig. 3 [7]

In the case of the wavelet transform, the important fact is that the real signal recorded by the measuring equipment is always matched to one wavelet of the whole set, with scale and translation 
factors changed, which results in unnecessary multiple descriptions of the same part of the signal.

\subsection{Matching pursuit algorithm (MP)}

Non-stationary signals are characterized by very high volatility, and concise representation of them can be achieved by describing them through the selection of a function from a very large redundant set, called a dictionary, from which functions best reflect the characteristics of the signal [21].

In order to capture the most relevant and reliable data on the structure of the recorded vibrations, the Matching Pursuit (MP) can be applied, a method of time - frequency analysis $[21,22]$, based on an iterative process of matching a whole set of functions to a signal. However, after each stage of approximation, the signal portion matched to a given function is subtracted, and the new functions are compared to the rest of the signal. This enables avoiding unnecessary multiple descriptions of the same part of the signal.

The MP algorithm involves the decomposition of a signal with time - frequency functions called atoms, selected from a dictionary $G=\left\{g_{1}(t), g_{2}(t), \ldots, g_{n}(t)\right\}$.

The concept of the atom should be defined as an elementary signal part whose aim is to best reflect the characteristics of the signal. The atom can be expressed as a single function window $g(t)$ by scaling a, translation b and frequency modulation $\xi$ (Eq. (1)) [21]:

$g_{I}(t)=\frac{1}{\sqrt{|a|}} \cdot g\left(\frac{t-b}{a}\right) \cdot e^{i \xi t}$,

where: $I$ - index defining the atom set of parameters, $I=(a, b, \xi), a$ - scale factor, $b$ - shift (translation) factor, $\xi$ - frequency modulation, $e^{i \xi t}-$ complex exponential function.

In practice, the Gabor dictionary is used, containing Gabor atoms, sine wave signals modulated by a Gaussian envelope (Fig. 8). Multiplying these two functions by themselves can result in a whole range of shapes, depending on their characteristics. The amplitudes are tuned in such a manner that each function (atom) has equal (unit) energy, as the scale product of the timeline of unit energy and the signal will be a direct measure of the share of the given structure in the signal energy.

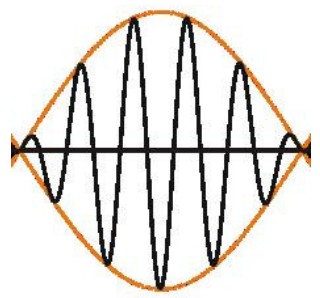

Fig. 8. Gabor atom scheme $[6,7]$

Regardless of the variety of shapes of Gabor atoms, the argument for their use is that they provide the best, optimal time - frequency resolution. In addition, the MP algorithm may be used with any dictionary containing a sufficient number of functions, so as to reproduce any signal.

With a dictionary comprising a variety of Gabor atoms, one should consider how to use them to make the process of approximation as effective as possible. One can try to use the products of all atoms and signals but, due to the large resources of the vocabulary, the most representative functions need to be selected.

For example, Fig. 9 shows four Gabor atoms, matched to specific fragments of the recorded signal $[7,17,18]$. Atoms numbered $0,3,6$ and 7 , shown in the Figure, successively explained $36.6 \%, 4.61 \%, 3.20 \%$ and $2.93 \%$ of the signal. Other atoms, matched to the signal $(1,2,4,5,8$ 
and 9) were deliberately omitted in order not to obscure the drawing.

As the signal decomposition progresses, the number of matching atoms is used and the residues contain less and less energy. The process of approximation of atoms to the signal does not last forever. The MP algorithm is stopped when approximately $95 \%$ of the energy of the signal is explained (or after a set number of repetitions), and the remainder is treated as an error of approximation (e.g. the noise in the signal).

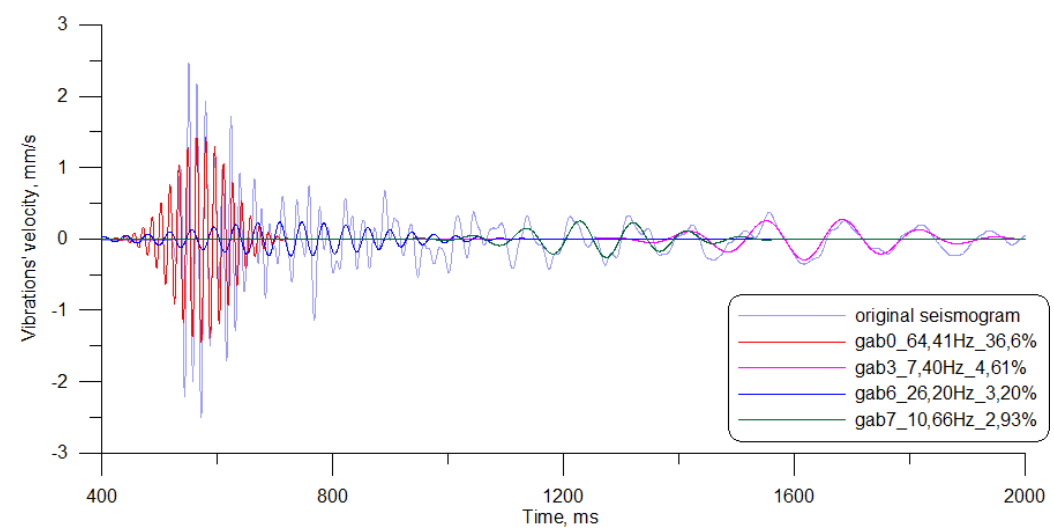

Fig. 9. Gabor atoms approximated to the scenario presented in Fig. 3 [7]

Results of the analysis using the method of matching pursuit are presented in a table (Table 1), including characteristics of individual parameters of Gabor atoms. On this basis, one can make a very thorough time - frequency analysis of a signal and, of particular importance, describe the share of individual Gabor atoms in the construction of the vibration energy image.

Table 1. The Matching Pursuit analysis, table of results

\begin{tabular}{|c|c|c|c|c|c|c|c|}
\hline $\begin{array}{c}\text { Gabor } \\
\text { atom no. }\end{array}$ & $\begin{array}{c}\text { Time, } \\
\mathrm{s}\end{array}$ & Position & $\begin{array}{c}\text { The amplitude of the } \\
\text { Gabor atom signal, } \\
\mathrm{mm} / \mathrm{s}\end{array}$ & Scale & $\begin{array}{c}\text { The length of } \\
\text { the Gabor } \\
\text { atom, } \mathrm{s}\end{array}$ & $\begin{array}{c}\text { Frequency, } \\
\mathrm{Hz}\end{array}$ & $\begin{array}{c}\text { The energy of the } \\
\text { Gabor atom } \\
\text { signal, } \mathrm{mm}^{2} / \mathrm{s}^{2}\end{array}$ \\
\hline 0 & 1.257 & 2515 & 0.891 & 1037 & 0.49 & 7.8 & 145.3 \\
\hline 1 & 1.352 & 2704 & 0.398 & 1492 & 0.70 & 10.5 & 41.7 \\
\hline 2 & 0.950 & 1900 & 0.884 & 297 & 0.14 & 18.1 & 41.0 \\
\hline 3 & 1.108 & 2216 & 0.505 & 657 & 0.31 & 4.5 & 29.5 \\
\hline
\end{tabular}

For example, based on Table 1, we obtain the following information about parameters:

Gabor atom no. - determines subsequent atoms participating in the reconstruction of the signal and explaining certain percentage of its energy. The order of the atoms is determined by the energy value represented by the given atom.

Time - specifies the location of the Gabor atom center on the timeline.

Position - determines the position of the Gabor atom center expressed in the number of the signal samples.

Scale (scale factor) - results in the change of the duration of the Gabor atom, its compressions or tensions.

The length of the Gabor atom - specifies the width of the atom in the time domain and depends on the number of samples and sampling frequency.

Frequency - the Gabor function modulation frequency.

Gabor atom signal energy - using this parameter, the energy value represented by a window function (Gabor atom), as the square of its amplitude, is specified.

For a better illustration, some parameters are shown as exemplified by a zero atom in Table 1 (Fig. 10). 

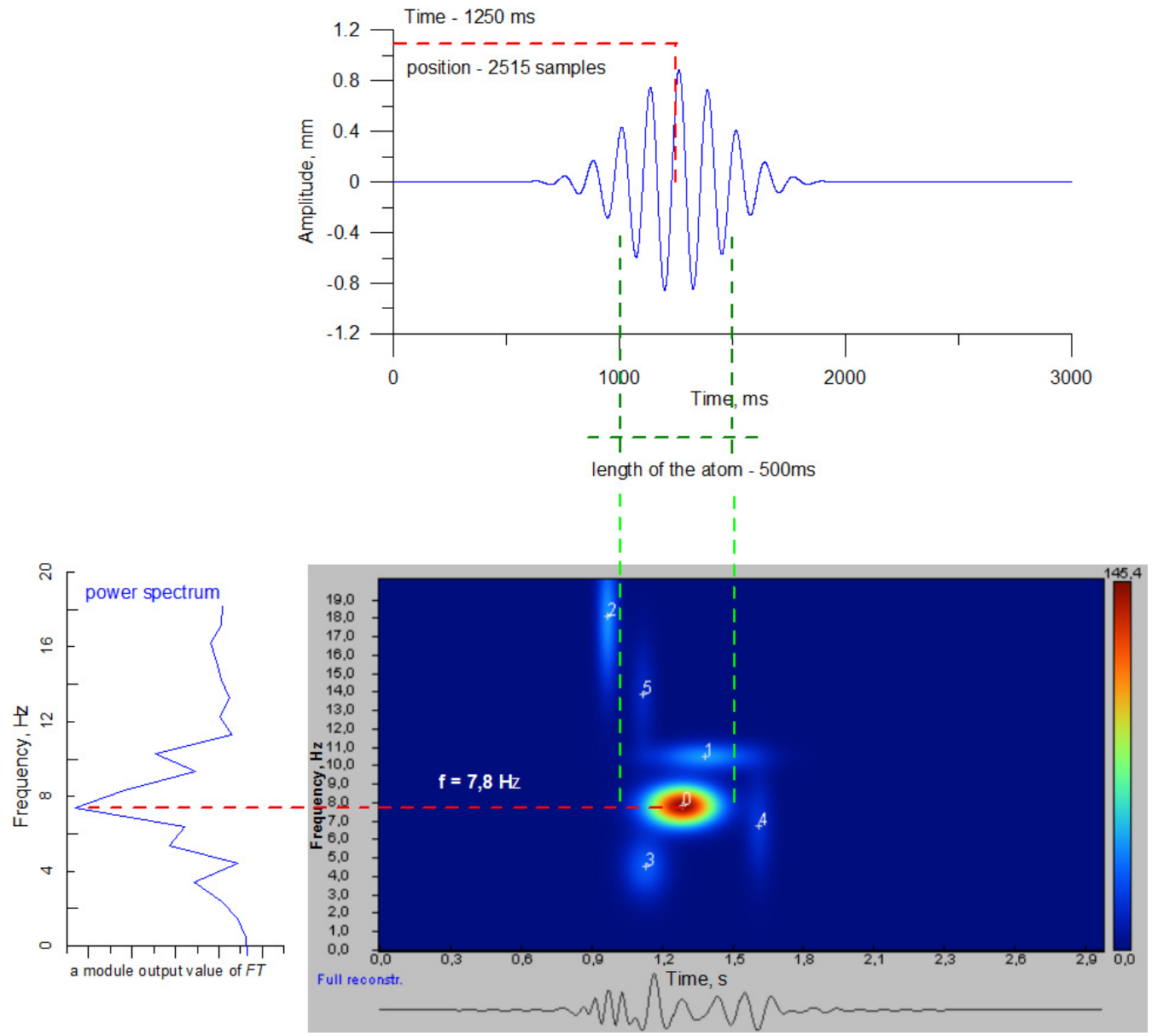

Fig. 10. Graphical representation of certain Gabor atom parameters [7]

In addition, the results of the Matching Pursuit are illustrated as the distribution of the energy density obtained with the Wigner distribution, also called the Wigner map (Fig. 11).

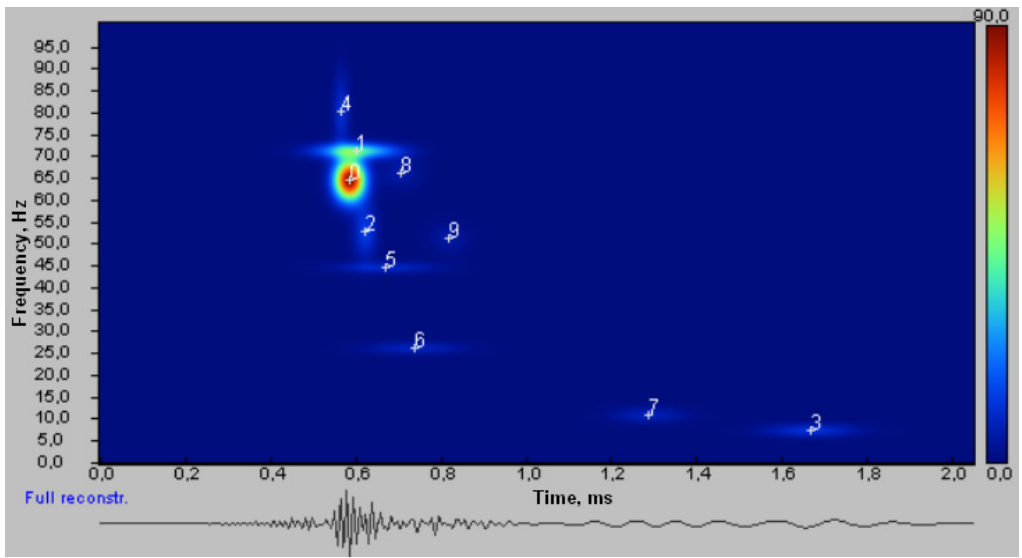

Fig. 11. The Wigner map for the timeline in Fig. 3 [7]

The Wigner map shows Gabor atoms (in the form of colored spots - "blobs") that matched to 
the actual signal, reconstituted it and explained approximately $95 \%$ of its energy. As seen in Fig. 11, Gabor atoms make it possible to timeline the characteristic frequencies, important in the signal construction. The color intensity of each atom is the energy density, as represented in various frequency bands. The sum of time - frequency atoms matched gives the signal energy, in accordance with the principle of energy conservation.

In order to demonstrate the precision of the reconstruction of the actual signal by Gabor atoms, Fig. 12 shows Fig. 3's (blue) actual vibration waveform and the reconstructed signal (red) on it. As it can be seen, the differences are minor, especially in the initial phase of the signal excitation, which indicates a very precise matching of time - frequency atoms to the signal and exact reconstruction of its features. It should be noted that the recorded signals may be interfered with (e.g. with noise), resulting in the formation of certain differences between the real signal and the reconstructed signal.

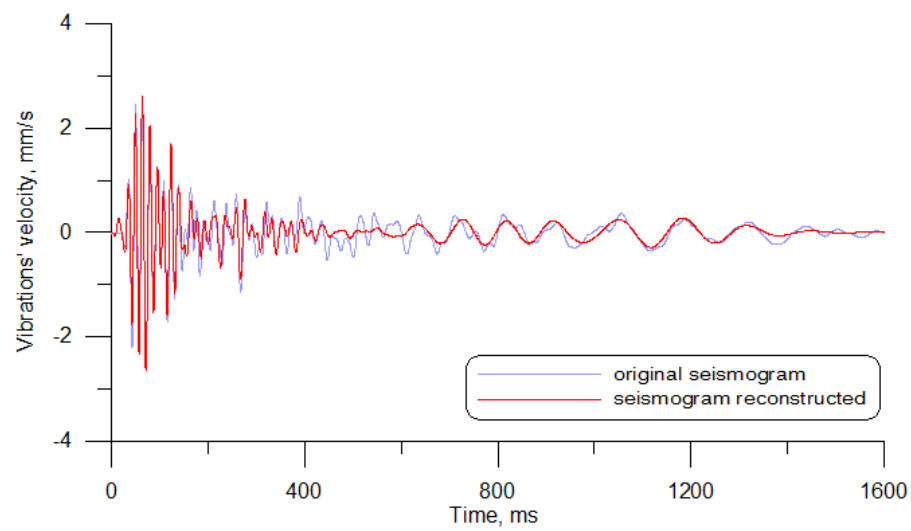

Fig. 12. A comparison of the Fig. 2 actual timeline and its reconstruction by Gabor atoms [7]

The structure analysis of signals can employ several methods, and selecting the most suitable one remains an open issue. However, you should consider which one is best from the perspective of a good characterization of the features of the recorded vibrations. In the case of the Fourier transform (FT), there is a possibility to analyze the signal in one of the two domains - the time domain or the frequency domain, but never in both, which does not enable obtaining information on the full vibration image. However, it precisely describes stationary signals in which there is a small range of frequencies. Using two new and popular methods of signal analysis, i.e. the shorttime Fourier transform (STFT) and the wavelet transform (CWT), there is a possibility of describing signal features using a set of functions matched to them. The wavelet transform includes functions called wavelets, and the STFT includes window functions used for cutting signal fragments, and their Fourier transforms are calculated. Use of these methods results in a vibration image on a time - frequency plane, but they have their drawbacks, hindering the precise characterization of the structure of vibrations. In the case of the STFT, there is a problem of selecting the optimum width of the window, selected by trial and error, while, in the case of the wavelet transform, it may be difficult to accurately describe, on a selected wavelet, both lower and higher frequencies. In addition, the original signal is matched to the same wavelet, despite the change of its parameters, resulting in the possibility of some portions of the signal being unnecessarily repeatedly described.

With regard to vibrations recorded while blasting, the choice of the Matching Pursuit algorithm, providing good time - frequency resolution and, at the same time, characterizing the energetic image of signals, is very important. For example, based on the results obtained, it is possible to conduct analyses enabling identification of the differences in ground quality under buildings, at the source of vibrations or the quality of the center where blasting is done. 


\section{Conclusions}

The ongoing technological progress in blasting works in open-cast mining definitely increases the possibility of controlling undesirable seismic effects. Vibrations induced at detonation can damage buildings in the environment. Taking up measures aimed at minimizing this impact should include careful recognition of the structure of vibrations and determination of which parameters of the vibration source can be crucial to reducing the incidence and intensity of impact or increased suppression at the transfer from the ground to the foundation.

The article discusses the various methods of signal analysis in order to present their capabilities and limitations in the context of their application to the analysis of vibration induced by blasting works.

Description of vibration using only one parameter for example a maximum value or frequency, without the knowledge of the interrelationship between these parameters is insufficient.

It is necessary to accurately recognize signals in terms of their structure and the possibility of interfering with them. Controlling structure vibrations can be an essential element enabling minimizing the impact of blasting on the environment.

Thus, for the analysis of vibrations induced by blasting works to be used time-frequency methods, which significantly improves the description of vibrations - both the wavelet analysis and the Matching Pursuit analysis identify the complex structure of pulsed signals with regard to the duration and energy of individual components.

In order to conclude that an impact is in the form of paraseismic vibration is imperceptible, noticeable, or harmful to the building object structure of the time - frequency vibration must be correlated with the appropriate scale used in particular country for this purpose. From the point of view of Polish scale SWD-I, the most unfavorable frequency is $25 \mathrm{~Hz}$, therefore blasting should be performed to the extent of mentioned frequency with the smallest amplitude of the recorded vibration.

\section{References}

[1] Zembaty $\mathbf{Z}$. The role of velocity in the intensity evaluation of the mining tremors. Tremors mining characteristics of vibration parameters and criteria for assessing the impact on buildings. Scientific Papers GIG - Mining and Environment, Katowice, 2010.

[2] Evaluation of the Harmfulness of Vibration Transmitted by the Ground on Buildings. PN-B02170:1985.

[3] Winzer J. Contribution to the discussion about manners of minimization of the impact of blasting works on buildings structures in the environment. Conference - Blasting Engineering in Mining and Construction, Ustron, 2013, p. 360-361.

[4] Siskind D. E., Stagg M. S., Kopp J. W., Dowding C. H. RI 8507 - Structure Response and Damage Produced by Vibration from Surface Mine Blasting. Bureau of Mines USA, 1980.

[5] Modrzejewski Sz. Principles of millisecond delay tome selection in rock mining. Surface Mining, Vol. 48, Issues 3-4, 2006, p. 153-157.

[6] Pyra J. Influence of a Millisecond Delay on the Vibration Response Spectrum Which is Induced by Detonation of Explosives. Doctoral Dissertation, Mining and Geo-Engineering Faculty, AGH University of Science and Technology, Cracow, 2011.

[7] Soltys A. Analysis of the Vibrations Impact on the Environment Induced by Blasting Using the Method of Matching Pursuit. Doctoral Dissertation - Monograph, AGH University of Science and Technology, Cracow, 2015.

[8] Winzer J. The Impact of Millisecond Delays in the Conducting Exploitation Blasting at the Frequency Vibration Characteristics of Land and Buildings on the Example of Deposits of Gypsum. Doctoral Dissertation, AGH University of Science and Technology, Cracow, 2004.

[9] Ozimek E. The Theoretical Basis of Spectral Analysis of Signals. PWN, Poznan, 1985.

[10] Modrzejewski Sz. Projecting the environmental impact of shot-blasting work in open-pit mining. Surface Mining, Vol. 46, Issues 5-6, 2004, p. 35-42.

[11] Modrzejewski Sz. Vibration velocity taken as paraseismic propagation indicator: invitation to discussion. Mining and Geoengineering, Vol. 28, Issue 3/1, 2004, p. 361-372. 
[12] Oloffson S. O. Applied Explosives Technology for Construction and Mining. Second Edition, Nora Boktryckeri AB, Sweden, 1988.

[13] Siskind D. E. Noise and Vibrations in Residential Structures from Quarry Production Blasting: Measurements at Six Sites in Illinois. University of Michigan Library, 1979.

[14] Dubiński J., Mutke G., Jaśkiewicz K., Lurka A. Intensity Scale of Mining GSI-2004 to evaluate/evaluating the effects of vibrations caused by mining tremors in the area LGOM - origins, the scale and verification. Seminar "Experiences with the use of scale GSI-2004", Cracow, 2008.

[15] Marcak H. Parameters of a ground motion model induced by mining exploitation. Archives of Mining Science, Vol. 53, Issue 3, 2008, p. 335-348.

[16] Marcak H., Kłosiński J. Frequency-time characteristics of seismic oscillations caused by mining tremor. Archives of Mining Science, Vol. 49, Issue 2, 2004, p. 193-204.

[17] Soltys A. The results of the matching pursuit analysis to assess the influence of vibrations on objects. Mining and Geoengineering, Vol. 34, Issue 2, 2010, p. 573-584.

[18] Soltys A., Pyra J. Application of time - frequency methods for analysis of vibrations induced by blasting in open cast mining. Work Safety and Environmental Protection in Mining, Vol. 5, 2010, p. 29-39.

[19] Tariova A. Signal Processing Systems. Wavelet analysis. Teaching and Learning Materials, West Pomeranian University of Technology Szczecin, 2007.

[20] Kwaśniewski J. Application of the wavelet analysis to inspection of compact ropes using a high efficiency device. Achieves of Mining Science, Vol. 58, Issue 1, 2013, p. 159-164.

[21] Durka P. J. Between Time and Frequency: Elements of Modern Signal Analysis. Script for the Lecture: Analysis of the Signal, Warsaw, 2004.

[22] Mallat S., Zhang Z. Matching pursuit with time - frequency dictionaries. IEEE Transactions on Signal Processing, Vol. 41, Issue 12, 1993, p. 3397-3415.

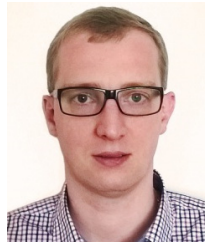

Józef Pyra received Ph.D. degree in AGH University of Science and Technology, Cracow, Poland, in 2011. Now he works at AGH University of Science and Technology. Since 2014 he is the head chief of the AGH Central Laboratory of Explosives and Blasting Works in Regulice City. His current research interests include explosives, blasting works, impact of vibration on the environment and analysis of signal. He is the author of more than 300 industrial reports for open pit and underground mines.

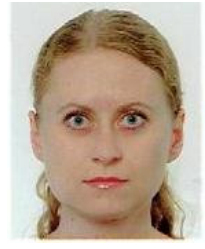

Anna Soltys received Ph.D. degree in AGH University of Science and Technology, Cracow, Poland, in 2011. Now she works at AGH University of Science and Technology. Her current research interests include explosives, blasting works, impact of vibration on the environment and analysis of signal. She is the author of more than 300 industrial reports for open pit and underground mines. She is also a member of the Association of Polish Explosives Engineers, which belongs to the European Federation of Explosives Engineers (EFFE). 